Gut, 1986, 27, 926-928

\title{
Late outcome of bleeding gastric ulcers
}

\author{
H L SMART AND M J S LANGMAN \\ From the Department of Therapeutics, University Hospital, Nottingham
}

SUMmaRY The subsequent progress of 152 patients treated medically in the Nottingham hospitals for bleeding gastric ulceration was analysed four to eight years later by the use of life tables. The overall cumulative rate of ulcer haemorrhage, perforation or related death was found to be $19 \%$. Women had a greater rate than men (women $21 \%$, men $14 \%$ ) but this difference was not statistically significant. The risk of developing a recurrent complication fell with time in both groups. Women were older than men at the time of their index admission and women who suffered a late complication were significantly older than those remaining well at the end of the follow up period. No similar differences existed for men. The risk of having a late complication from a bleeding gastric ulcer compares favourably with that reported under similar circumstances for duodenal ulceration. Attempts to reduce the late complication rate should concentrate on those patients shown to be at a greater risk - that is, elderly women.

Bleeding gastric ulcers account for a fifth of admissions with haematemesis and melaena. ${ }^{1}$ The immediate prognosis is generally believed to be poorer than that of duodenal ulcer but there is no substantial body of data to demonstrate the late outcome. Interpretation of the results of the few available studies ${ }^{2-5}$ is hindered because patient numbers were small and life table methods were not used to combine sets of data obtained in patients followed for varying time periods.

We have therefore examined the frequency of recurrent gastrointestinal haemorrhage, perforation or related death in a large consecutive group of patients who were originally admitted with gastric ulcer bleeding and managed medically.

\section{Methods}

PATIENTS

Between June 1975 and December 1979 over 1300 patients were admitted to the City and General Hospitals in Nottingham with acute upper gastrointestinal haemorrhage. Two hundred and ninety of these patients were diagnosed either endoscopically or radiologically as having gastric ulceration at the time of admission (index admission). Excluding patients who were subsequently treated surgically

Address for correspondence: Professor M J S Langman. Department of Therapeutics, Floor C, South Block, Unversity Hospital, Nottingham NG7 2UH.

Received for publication 21 November 1985
(89), had an associated upper gastrointestinal lesion - for example duodenal ulcer (27), died on index admission (20) or developed a gastric cancer within one year of index admission (two), a total of 152 patients were available for follow up.

The frequency of recurrent ulcer complications (bleeding or perforation) requiring readmission to hospital after initial discharge was then studied for the period from the index admission until 1 April 1984. Details were therefore obtained for varying periods, with a minimum of 52 months and a maximum of 106 months. Information regarding the patients and progress was obtained by reference to hospital case notes, supplemented by questionnaires completed by the patients' general practitioner. In those patients who had died details of the cause of death was obtained from death certificates.

The data were analysed by the construction of life tables to show the risk of recurrent haemorrhage, perforation, or ulcer related death in those at risk. Patients who died from non-ulcer related causes and those in whom incomplete data were available were regarded as censored observations at the time of death or last clinic attendance respectively and they were counted as being followed for half the interval in which the censoring took place. In constructing life tables six monthly intervals were used and the probability of suffering a complication in that interval was calculated by the number of events (bleeding or perforation) during the interval divided by the number of patients under follow up (the number alive at the beginning of the interval minus 
one half of the number of whose data were censored during the interval). The cumulative probability of bleeding or perforation was computed as the product of the probabilities in each interval. Tables were constructed for men, women and all subjects. Comparisons of data were carried out by Log-rank testing. ${ }^{6}$

\section{Results}

Seventy five $(49 \%)$ of the 152 patients who left hospital were still alive and free from ulcer complications after a median interval of six years (range four to eight years). Thirty nine $(26 \%)$ had died of non-ulcer disease, non-gastric malignancy (12 deaths) being the commonest cause. One died of gastric cancer 18 months after index admission (Table). Four, all women had died after further gastric ulcer bleeding (three) or perforation (one), with one patient dying after non-gastric ulcer bleeding; in addition $25(16 \%)$ of patients were admitted to hospital with further episodes of ulcer bleeding. The remaining eight patients $(5 \%)$ were lost to follow up.

The Figure shows the risk, by life table analysis, of developing a late complication of gastric ulceration in six monthly intervals over the ensuing eight years. By this method the cumulative risk of such a late event was $19 \%$ overall. In general the risk of having a late ulcer complication decreased with time: risk in the first year $7 \%$, after the first two years $11 \%$, and $16 \%$, after five years. No ulcer complications occurred after five and a half years in any of the subjects studied.

Table Causes of death in patients during the follow up period.

\begin{tabular}{llc}
\hline Cause of death & Men & Women \\
\hline Ulcer related & & \\
$\quad$ Bleeding gastric ulcer & 0 & 3 \\
$\quad$ Perforated gastric ulcer & 0 & 1 \\
Non-ulcer related & & \\
Malignancy & $7^{*}$ & $6 \dagger$ \\
Cerebrovascular disease & 2 & 6 \\
Myocardial infarction & 4 & 2 \\
Cardiac failure & 1 & 3 \\
Aortic dissection & 1 & 0 \\
Bronchopneumonia & 1 & 1 \\
Chronic bronchitis & 1 & 1 \\
Epistaxis & 0 & 1 \\
Suicide & 1 & 0 \\
Perforated duodenal ulcer & 0 & 1 \\
Not known & 0 & 1 \\
& 18 & 26 \\
\hline
\end{tabular}

*prostate 3 , bronchus, rectum, stomach, myeloma 1 tbreast 2 , rectum, bladder, vulva, leukaemia 1

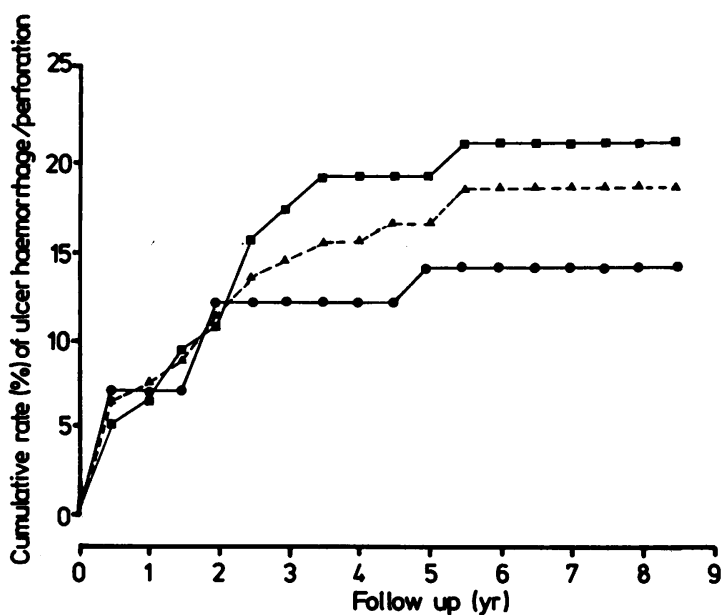

Figure Late outcome of 152 patients admitted with a gastric ulcer bleed. All cases ( $\mathbf{\Delta}---\mathbf{\Delta})$, women

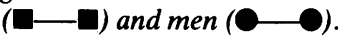

Taken overall women were significantly older $(p<0.0005)$ than men at the date of their index admissions (mean ages 65 years and 57 years respectively) and were more likely to suffer from a late complication $(21 \%$ and $14 \%$ respectively) although the difference was not significant statistically $(\mathrm{p}=0.5$ by log-rank testing). Women who suffered from recurrent bleeding or perforation were, however, significantly older $(p<0.025)$ than those who remained well at the end of the follow up period (mean ages 75 years and 65 years respectively) although no similar trend was detectable in men.

\section{Discussion}

We have shown, by applying life table analysis to a large consecutive group of patients admitted with bleeding from gastric ulceration, that almost one fifth of these can be expected to either rebleed or perforate during the ensuing years. Studies of the late outcome of bleeding gastric ulcers have generally been small and incomplete. ${ }^{2-5}$ 7-9 As a consequence life table analysis has not been undertaken and therefore direct comparison with our present study is impossible, but recurrent haemorrhage or perforation was reported in between $9 \%{ }^{4}$ and $67 \%^{5}$ of patients during the follow up period.

The cumulative risk or rebleeding in our gastric ulcer patients is similar to, although slightly less than that obtained in patients admitted to the Central Middlesex Hospital with duodenal ulcer bleeding ${ }^{10}$ where the cumulative risk of a recurrent ulcer bleed or perforation was $23 \%$. It would appear therefore 
that patients with a gastric ulcer bleed have a no less favourable prognosis in respect to late haemorrhage and perforation than those presenting with duodenal ulceration.

In this retrospective analysis we have not been able to study such factors as treatment, smoking, alcohol and non-steroidal intake which may be related to recurrent ulcer problems. Although women with recurrent bleeding were particularly likely to be elderly no similar trend was evident in men and we know of no similar pattern which has been detected elsewhere.

\section{References}

1 Dronfield MW, Langman MJS, Atkinson M et al. Outcome of endoscopy and barium radiography for acute upper gastrointestinal bleeding: controlled trial in 1037 patients. $\mathrm{Br}$ Med J 1982; 1: 545-8.

2 Chinn AB, Littell AS, Badger GF, Beams AJ. Acute haemorrhage from peptic ulcer: a follow up study of 310 patients. $N$ Engl J Med 1956; 255: 973-8.
3 Arias IM, Zamcheck N, Thrower WB. Recurrence of haemorrhage from medically treated gastric ulcers: four to eight year follow up of 47 patients. Arch Intern Med 1958; 101: 369-76.

4 Grace NJ, Mitty WF. Does subtotal gastrectomy in bleeding peptic ulcer prevent recurrence of bleeding? Am J Dig Dis 1962; 7: 69-74.

5 Leape LL, Welch CE. Late prognosis of patients with upper gastrointestinal hemorrhage. Am J Surg 1964; 107: 297-305.

6 Coldman AJ, Elwood JM. Examining survival data. Can Med Assoc J 1979; 121: 1065-71.

7 Flood CA, Hennig GC. Recurrence in gastric ulcer under medical management. Gastroenterology 1950; 16: $57-72$.

8 Borland JL, Hancock WR, Borland JL. Recurrent upper gastrointestinal hemorrhage in peptic ulcer. Gastroenterology 1967; 52: 631-7.

9 Swynnerton BF, Tanner NC. Chronic gastric ulcer: a comparison between a gastroscopically controlled series treated medically and a series treated by surgery. Br Med J 1953; 11: 841-7.

10 Harvey RF, Langman MJS. The late results of medical and surgical treatment for bleeding duodenal ulcer. $Q J$ Med 1970; 34: 539-47. 